\title{
Two different approaches for creating a prescribed opposed-flow velocity field for flame spread experiments
}

\author{
Luca Carmignani $^{1, a}$, Greg Celniker ${ }^{2}$, Kyle Bussett ${ }^{2}$, Christopher Paolini ${ }^{2}$ and Subrata Bhattacharjee ${ }^{2}$ \\ ${ }^{I}$ Department of Industrial and Civil Engineering, University of Pisa, Pisa, Italy \\ ${ }^{2}$ Department of Mechanical Engineering, San Diego State University, San Diego, California, USA
}

\begin{abstract}
Opposed-flow flame spread over solid fuels is a fundamental area of research in fire science. Typically combustion wind tunnels are used to generate the opposing flow of oxidizer against which a laminar flame spread occurs along the fuel samples. The spreading flame is generally embedded in a laminar boundary layer, which interacts with the strong buoyancy-induced flow to affect the mechanism of flame spread. In this work, two different approaches for creating the opposed-flow are compared. In the first approach, a vertical combustion tunnel is used where a thin fuel sample, thin acrylic or ashless filter paper, is held vertically along the axis of the test-section with the airflow controlled by controlling the duty cycles of four fans. As the sample is ignited, a flame spreads downward in a steady manner along a developing boundary layer. In the second approach, the sample is held in a movable cart placed in an eight-meter tall vertical chamber filled with air. As the sample is ignited, the cart is moved downward (through a remote-controlled mechanism) at a prescribed velocity. The results from the two approaches are compared to establish the boundary layer effect on flame spread over thin fuels.
\end{abstract}

\section{Introduction}

The term "flame spread" indicates the rate of flame movement across a fuel surface that typically has small scales (on the order of centimeters). Much effort has been spent to obtain a better understanding of flame spread rate in different conditions, especially with an opposing flow, due to the fundamental nature of the problem and its implications for fire safety, [1-4]. However, in literature, there are not many studies on the influence of the aerodynamic boundary layer on the flame spread, [5]. To investigate the importance of this aspect, and particularly its effect on the flame extinction, two different apparatuses have been built at San Diego State University (SDSU), and they are presented in this work.

The purpose of the two apparatuses, named "Flame Tower" and "Flame Tunnel", is the same: generate an opposing flow in the velocity range of $0-100 \mathrm{~cm} / \mathrm{s}$. The working concept is different: in the Flame Tower the sample is placed in a cart (called FlameTracker) which is moving inside the tower, while in the Flame Tunnel, the sample is fixed and the flow is generated by four fans.

The Flame Tower is eight meters tall, and the cart can reach the maximum velocity of $120 \mathrm{~cm} / \mathrm{s}$, with a maximum acceleration of $55 \mathrm{~cm} / \mathrm{s}^{2}$ (over this acceleration the flame extinguishes before reaching the desired velocity). The two inputs for the software that controls the cart motion are velocity and acceleration. In addition the width of the fuel sample can also be selected. Experiments are video-recorded, and then analyzed with an image software analyzer (Spotlight) to extract the needed data.

The Flame Tunnel also allows for the sample width to be chosen, but the input is the rotational speed of the fans. Sensors placed at the bottom of the sample holder are used to determine air velocity, and the desired velocity can be established in one or two minutes. The maximum reliable velocity obtainable in the tunnel is 100 $\mathrm{cm} / \mathrm{s}$, but in the future, new calibrations could increase this limit.

The design of the Flame Tunnel refers to the one introduced by $\mathrm{T}$. Hirano, for his experiments on flame spread rate, [4]. More recent advancement in technology have lowered the cost and effort required to construct such a device. As in the Flame Tower, experiments are video-recorded and then analyzed with Spotlight.

The flame leading edge velocity is close to the one shown by the sensors in both cases, but not equal, due to the flow interaction with the cart and the walls (for the Flame Tower) and the walls (for the Flame Tunnel). Regarding the Flame Tower, this difference has been proven to be small, both computationally and experimentally, [6]. The difference in leading edge velocity in the case of Flame Tunnel is also small, and

a luca.carmignani@icloud.com 
that will be proven in this paper. At the end of this work are the results from flame spread rate experiments, which were obtained using filter paper. They are shown with an opposing flow velocity of 30 and $60 \mathrm{~cm} / \mathrm{s}$ and are consistent for the two different approaches.

\section{Flame Tower calibration}

The Flame Tower allows the translational motion of a cart to be set to a desired velocity along a vertical rail.

The software control is realized through a Matlab application we developed, and it runs in a PC connected to the tower. This software directly controls a Parker COMPUMOTOR AX indexer that drives a Parker COMPUMOTOR Model M106-178 DC motor mounted at the top of the Flame Tower. The indexer also controls position, velocity, and acceleration of the cart, [6,7].

The purpose of the velocity control mechanism is to ensure that the cart velocity is constant through the entire length of travel and that the commanded velocity matches the actual cart velocity. These characteristics are important because oscillations in cart velocity cause variations in the oxidizer velocity seen by a flame propagating along a sample of fuel placed on a sample holder, which is fixed to the cart.

Velocity calibration was accomplished with postprocessing video analysis on a frame by frame basis. First measuring tape was placed along the vertical rail inside the tower. Then a Casio Exilim EX-FH20 camera was mounted on the carriage pointing the rail. The system was set with an acceleration of $100 \mathrm{~cm} / \mathrm{s}^{2}$, which was the highest reliable acceleration and the scale was filmed at 420 FPS at various velocities. The videos from the camera were then imported into editing software, where a line was overlaid over the videos to provide a reference point. The beginning and ending points of the videos were cut just as the desired tick mark passed the reference line, and then the number of frames and the travelled distance were logged. Travel time was obtained dividing the number of frames by 420 , and the following division of the travel distance by the time gave the cart velocity in $\mathrm{cm} / \mathrm{s}$. Obtained results of the video calibration are shown in Figure 1.

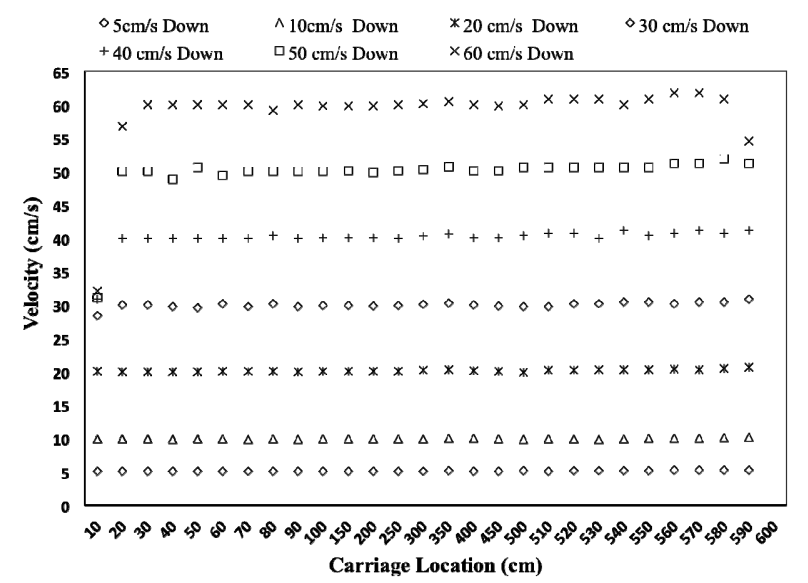

Figure 1. Measurements of cart velocity from video analysis.
This is the procedure followed to calibrate the cart velocity, but nothing about the actual velocity seen by the flame has been said. Velocity "experienced" by the flame depends on the cross section of the cart and on the flow interaction between the tower structure (walls, rail and counterweight conduct) and the cart.

For the current cart, named FlameTracker3, it was assumed that the velocity seen by the flame was very close to the one of the cart itself, neglecting any flow interaction. This assumption was suggested after the study on the previous carts (FlameTrackerl and FlameTracker2), [6,7]; it has been proven in fact that the ratio between the velocity seen by the flame and the one of the cart was about 1.05 .

\section{Flame Tunnel sensors calibration}

The Flame Tunnel velocity control is based on the communication between a running code in Matlab and an Arduino micro-controller (MCU).

To detect the internal flow of the Flame Tunnel two Resistance Thermal Detector (RTD) sensors were placed right below the sample holder: the velocity detected by sensors is very close to the actual one seen by the flame at sample leading edge. This couple of sensors guarantees good accuracy and precision especially for low velocities, which is a requirement for experiments carried on with Flame Tunnel.

The sensors are connected in a Wheatstone bridge: one of the RTDs is actively heated, so the resistance varies in accordance with the convection associated with air flow, while the other one is not heated (no response to changing flow velocity). The voltage difference given by the Wheatstone bridge is amplified and collected by the MCU, which converts the signal in a number from 0 to 1023 (ten bit) and communicates with a program developed in Matlab that converts the voltage signal into velocity. The Wheatstone bridge ensures that velocity can be determined regardless of small changes in room temperature.

RTDs were calibrated using the velocity-calibrated Flame Tower. An anemometer was placed on FlameTracker3 in place of the leading edge of the sample. The sensor used is an Omron D6F-W01A1 MEMS Flow Rate Sensor, which gives voltage output from 1.00 to $5.00 \mathrm{~V}$ in a velocity range from 0 to 100 $\mathrm{cm} / \mathrm{s}$. For velocity range from 0 to $40 \mathrm{~cm} / \mathrm{s}$ data were collected with $5 \mathrm{~cm} / \mathrm{s}$ steps, for the range from 40 to 100 $\mathrm{cm} / \mathrm{s}$ steps were $10 \mathrm{~cm} / \mathrm{s}$ (Figure 2).

The anemometer was then placed at the leading edge of the Flame Tunnel sample holder, and the fan speed was adjusted to reach voltage values as close as possible to the ones of the curve in Figure 2 (for a better comparison).

Using collected data and the trend of Figure 2 to compute velocity values from voltage output, a curve for RTD values as function of velocity was obtained (Figure $3)$. 


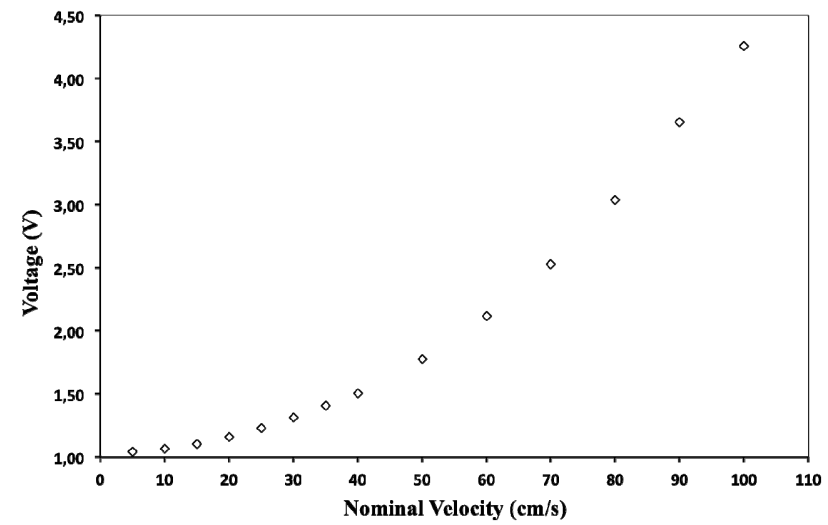

Figure 2. Anemometer output (voltage) as function of different velocities of FlameTracker3.

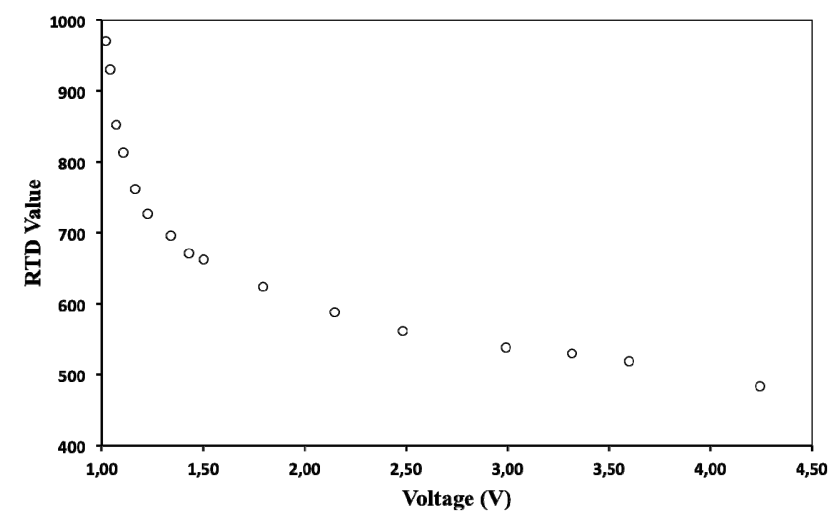

Figure 3. Data plot used for the calibration of RTDs in the Flame Tunnel.

A sixth-order polynomial data fit was extrapolated from the curve of Figure 3, and it was used for the code that runs in the Matlab code. For velocities over $100 \mathrm{~cm} / \mathrm{s}$ data from RTDs factory were taken as reference for a better understanding of sensors behavior.

To validate the new code additional tests were performed in order to compare RTDs and anemometer values.

\section{Simulations for flow interactions}

The Flame Tunnel geometry (with the sample holder) was modeled in SolidWorks and imported into ANSYS Fluent, where 2D flow simulations were performed to compare computational results against analytical and experimental.

Assuming a reference system with the y-direction orientated along the vertical direction of the tunnel and the $\mathrm{x}$-direction perpendicular to the sample surface, an accurate investigation of the flow behavior near the leading edge was realized using the MUSCL algorithm.

The result of the simulation performed with pressure at second order and momentum at the third order is shown in Figure 4, considering a uniform flow velocity of $40 \mathrm{~cm} / \mathrm{s}$ at the bottom of the tunnel.

Since the area ratio between the two cross-sections of the tunnel is four, the resulting flow near the sample from a simplified 1D analytical approach is about 160 $\mathrm{cm} / \mathrm{s}$, as confirmed by the simulations.

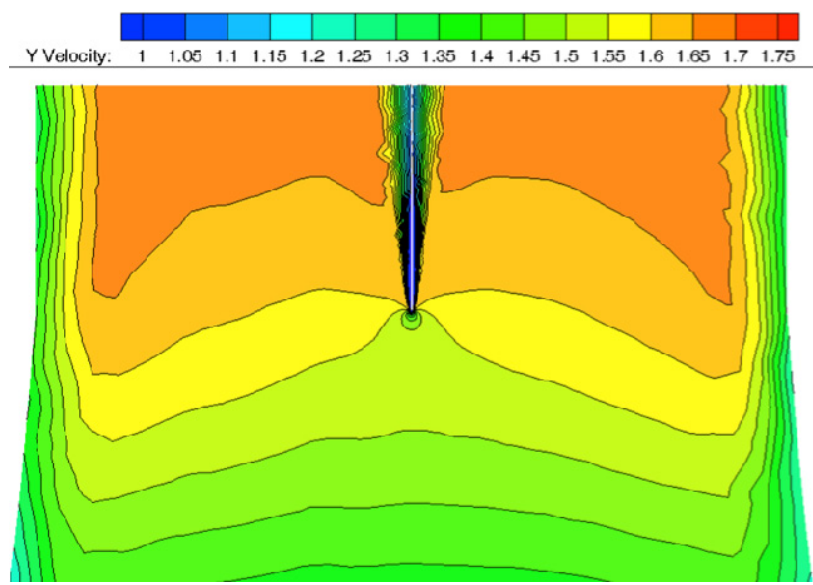

Figure 4. Pressure $2^{\text {nd }}$ order, momentum $3^{\text {rd }}$ order simulation of the flow inside the Flame Tunnel near the leading edge, using MUSCL algorithm.

The velocity profile close to the leading edge was further investigated to have a better understanding of the behavior of the velocity field in the y-direction. In Figure 5 are shown the velocity profiles for three different locations, at a distance of 5,10 and $20 \mathrm{~mm}$ from the leading edge in the positive y-direction. The graph axes are normalized: the $\mathrm{x}$-axis expresses the distance from the leading edge $(\mathrm{x})$ respect to the dimension of the tunnel (D), and the y-axis shows the flow velocity with respect to the velocity at the bottom of the tunnel (bottom velocity), $\mathrm{u}_{\infty}$. As previously stated, the expected ratio between the velocity seen by the flame and the one at the bottom was about four, and has been computationally confirmed.

Referring again to Figure 5, it can be noted that the velocity seen by the flame is actually very close to that of the flow field. Thus, the assumption that RTDs can sense a velocity very close to the one seen by the flame is reasonably satisfied, especially for low velocities.

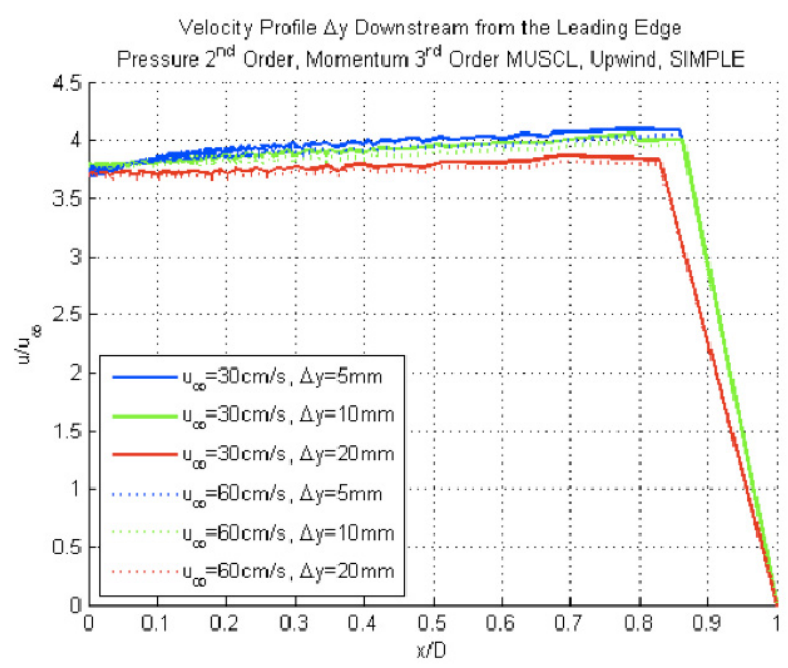

Figure 5. Velocity profile at three different locations near the leading edge, for two different velocities (represented velocity values are referring to the bottom velocity). 


\section{Flame spread comparison}

The procedure to obtain flame spread rate data from experiments with the Flame Tower and the Flame Tunnel is the same.

Once the recorded video of the experiments are imported into Spotlight, it is possible to track the flame leading edge location at different frames, which correspond to time steps. The collected data are then averaged to obtain the flame spread for every velocity.

For thin fuels and low velocities, the flame spread rate could be considered constant, because the flame is in quasi-steady state conditions. Hence, the curve representing the position of the flame versus time should be linear, and the value of flame spread rate is expressed by the slope, $[4,7]$.

Results of flame spread for filter paper samples were obtained in the presence of an opposing flow velocity of $30 \mathrm{~cm} / \mathrm{s}$ and $60 \mathrm{~cm} / \mathrm{s}$ both for the Flame Tunnel and the Flame Tower; sample width was $20 \mathrm{~mm}$ and the thickness $180 \mu \mathrm{m}$. With the assumptions of a thin fuel and low velocities the averaged data can be interpolated with a linear trend, obtaining constant flame spread rates (Table 1).
Table 1. Flame spread rate obtained with a linear interpolation of the position vs time diagram, expressed in $\mathrm{mm} / \mathrm{s}$.

\begin{tabular}{|c|c|c|}
\hline & Vel. $30 \mathrm{~cm} / \mathrm{s}$ & Vel. $60 \mathrm{~cm} / \mathrm{s}$ \\
\hline Flame Tower & 1.676 & 1.415 \\
\hline Flame Tunnel & 1.731 & 1.359 \\
\hline
\end{tabular}

The values of flame spread rate are consistent for the two cases; a little discrepancy was expected because of the two different lengths of the samples. In the Flame Tunnel, the sample is longer than in the Flame Tower, and the burning flame experiences different flow velocities while is going downward, due to the boundary layer effect, [5]. Recent studies demonstrated that the distance of the flame from the sample leading edge affects the velocity experienced by the flame leading edge, [8], and consequently the flame spread rate. Thus, for a given opposed-flow velocity, the flame spread rate depends on the burning part of the sample during the experiment. Since in the Flame Tower the quasi-steady state condition before the cart reaches the end of the track has a limited time, the burnt sample length interval is not exactly the same of the Flame Tunnel (Figure 6).
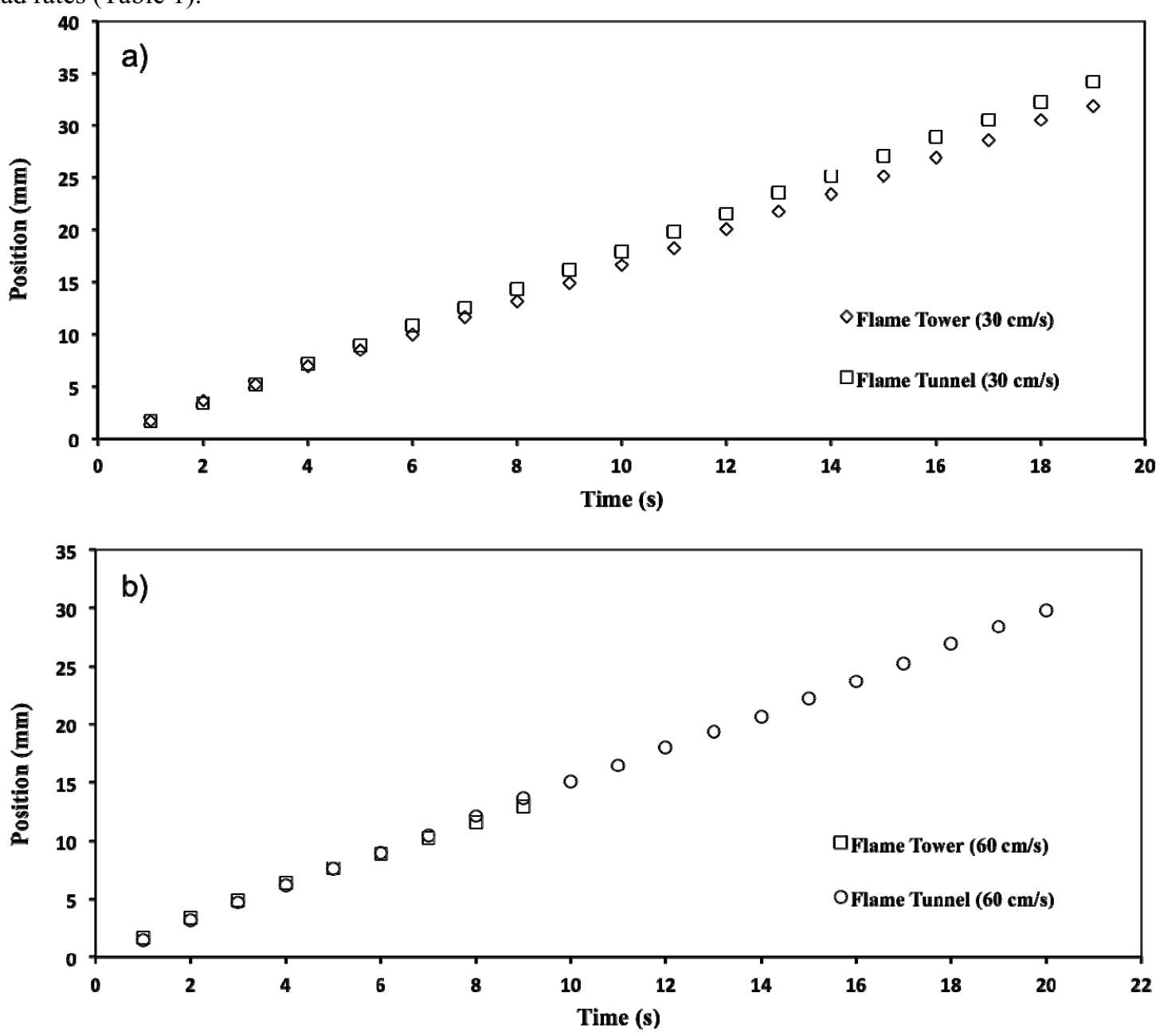

Figure 6. The slope of the lines indicates the flame spread rate. These graphs are obtained from experiments with an opposed-flow of: a) $30 \mathrm{~cm} / \mathrm{s}$; b) $60 \mathrm{~cm} / \mathrm{s}$. 
The results of the flame spread suggest that the calibration was done correctly, even though the error of the anemometer in full scale is relatively high and the detected flow velocity is not exactly the real one. Because of the fact that the two apparatuses were calibrated using the same values, the comparison of the obtained results is possible.

\section{Funding}

The research at SDSU is supported by a grant from NASA, with Dr. David Urban serving as the contract monitor.

\section{References}

1. I.S. Wichman, Prog. Energy Combust. Sci., pp 554-568 (1992)

2. S. Bhattacharjee, M.D. King, C. Paolini, Combust. Theory Modeling, pp 23-29 (2003)

3. S. Bhattacharjee, K.K. Bhaskaran, R.A. Altenkirch, Combust. Sci. and Tech. 100, pp 163-182 (1994)

4. T. Hirano, Proceedings $5^{\text {th }}$ AOSFST Newcastle, Australia, pp 40-53 (2001)

5. S. Bhattacharjee, W. Tran, M. Laue, C. Paolini, Y. Nakamura, $8^{\text {th }}$ U.S. National Combustion Meeting (2013)

6. C. Paolini, S. Bhattacharjee, W. Tran, F. Naib, F. Miller, $8^{\text {th }}$ U.S. National Combustion Meeting (2013)

7. N.K. Dalal, MS Thesis San Diego State University, Experimental approach in measuring flame spread rate (2011)

8. S. Bhattacharjee, R. Nagarkar, Y. Nakamura, Combust. Sci. Technol. 00, pp 1-13 (2014) 